ISSN: 2576-8875

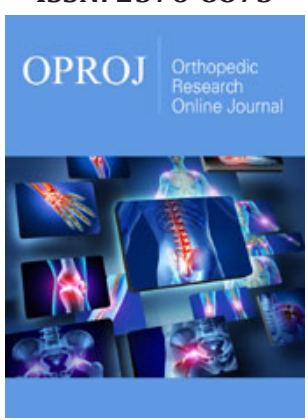

*Corresponding author: Brett Braly, The Spine Clinic of Oklahoma City, USA

Submission: 誡July 30, 2021

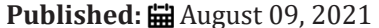

Volume 8 - Issue 4

How to cite this article: Brett Braly, Sameer Hasan, Ashley Mason, Abigail Lange. A Comparison of Intraoperative Fluoroscopic Radiation Emission Using ALARA Methods Versus ImageEnhancement Technology in SinglePosition Lateral Lumbar Surgery. Ortho Res Online J. 8(4). OPROJ. 000692. 2021. DOI: 10.31031/OPROJ.2021.08.000692

Copyright@: Brett Braly, This article is distributed under the terms of the Creative Commons Attribution 4.0 International License, which permits unrestricted use and redistribution provided that the original author and source are credited.

\section{A Comparison of Intraoperative Fluoroscopic Radiation Emission Using ALARA Methods Versus Image-Enhancement Technology in Single-Position Lateral Lumbar Surgery}

\author{
Brett Braly $^{1 *}$, Sameer Hasan ${ }^{2}$, Ashley Mason ${ }^{1}$ and Abigail Lange ${ }^{1}$ \\ ${ }^{1}$ The Spine Clinic of Oklahoma City, USA \\ ${ }^{2}$ Community Hospital, OKC, USA
}

\begin{abstract}
Background: Expanded interest in minimally invasive spine techniques has led to increased reliance on intraoperative imaging to guide surgical decision making. Attempts to minimize the amount of radiation by using the dosage 'as low as reasonably achievable' (ALARA) has proven acceptable in decreasing the risk to the surgeons and staff to a certain extent, though the reciprocal nature of radiation reduction and image usability (quality) challenge further expansion of ALARA in this setting. Emerging technologies propose to reduce the radiation requirements even further without compromising image quality. In this study, radiation emission and fluoroscopy time were evaluated when image-enhancing technology was applied to minimally invasive single-position lumbar surgery to further our understanding of how imageenhancement technology performs in comparison to established ALARA protocols.
\end{abstract}

Methods: This was a retrospective case-control study which in radiation emission and intra-operative fluoroscopy time was measured in patients undergoing lateral based, single-position surgery. From Oct 2018 through July 2019, 68 patients underwent one- or two-level single-position, lateral-based lumbar fusions by the same surgeon and operative team (including radiation technologist) at one of two hospitals. Both hospitals utilize an ALARA protocol in the operating rooms. One institution has acquired image-enhancing technology which aims to further reduce radiation emission and exposure while the other uses standard ALARA techniques. Intraoperative radiation data were retrospectively reviewed for comparison.

Results: Image-enhancing technology allowed for further reduction of radiation emission from the fluoroscope by $36 \%$ when compared to the current low-dose ALARA protocols. Operative workflow features within the image-enhancing system allowed for a reduction of overall fluoroscopic time requirements by $34 \%$, which aided in the overall $36 \%$ reduction in radiation emission.

Conclusion: Emerging image-enhancement technology allows for further reduction of radiation emission in the operating room by $36 \%$ when compared to current, low-dose protocols established by ALARA.

Keywords: Radiation; Emerging technology; Lateral-position surgery; Single-position surgery

\section{Introduction}

We present the following article/case in accordance with the STROBE reporting checklist.

Hospitals and outpatient surgical centers over the years have seen growing numbers in spine surgery as the population ages and concerns for prolonged physical function increases. One of the fastest growing fields in spine surgery has been the trend toward Minimally Invasive Spine (MIS) techniques. A primary benefit of MIS techniques is the ability to decrease the size of the incision and resultant reduction in procedural morbidity. However, a reduced incision size limits operative field visibility and creates an increased reliance on intraoperative fluoroscopy to guide surgical decision making during the procedure.

Orthopedic surgeons have long relied on intraoperative fluoroscopy for fracture fixation, and previous studies have suggested an increase in cancer rates among this population by 5 times [1]. Furthermore, spine surgeons are exposed to, on average, 10 to 20 times more 
radiation when compared to non-spine surgeons [2]. Increased radiation exposure during MIS Transforaminal Lumbar Interbody Fusion (TLIF) procedures have shown dosages averaging $138 \mathrm{mGy}$ for a single case, which is the equivalent radiation dosage of 14 lumbar CT scans [3].

Attempts to reduce the radiation exposure by decreasing the dosage used by the fluoroscope have been proven minimally effective at reducing levels to those needed to protect all intraoperative staff over the course of their careers [4]. Although low dose radiation settings within the fluoroscope are technically able to meet these goals, the results yield additional challenges that limits image quality for safe surgical decisions when instrumenting the spine. Many institutions already adhere to ALARA (as low as reasonably achievable) standards, but there was an opportunity to further decrease radiation emission while maintaining image quality through the use of image enhancement technology. The concern over long-term exposure to radiation in the workplace has driven many MIS surgeons to seek answers for radiation risk reduction. Reducing fluoroscopic radiation emission, using lower dosage and pulse images, decreases the clarity of intraoperative imaging and can significantly affect diagnostic utility for instrumentation and increases patient risk. Effectively, there is a minimum amount of radiation reduction that can be achieved while still producing images clear enough for accurate surgical decisions during the procedure.

The purpose of this study was to determine whether the amount of radiation emission and the surgical workflow differences between lumbar interbody fusion procedures performed with intraoperative fluoroscopic use at standard low-dose radiation levels versus lowerdose radiation levels through Image Enhancement Technology (IET). Image enhancement involves using a proprietary software to recreate images from low dose shots based on an initial scout image. In such the image produces appears as though it were taken at regular radiation dosage. The hypothesis was that low-dose fluoroscopic imaging with software-enhancement, as well as the incorporation of surgical workflow efficiency tools will, would lead to the reduction of radiation emission and fluoroscopic time during single-position lateral lumbar surgery.

\section{Methods}

A retrospective review of prospectively collected data was undertaken at a single site. Due to the retrospective nature of the study there was no need to account for failure of patient followup. All cases were completed by a single surgeon at two locations to allow for the different radiological viewing methods. Patients self-selected their hospital based solely on availability and location. From Oct 2018 through July 2019, a total of 68 patients were treated with either single-level or two-level lumbar interbody fusion. The standard low-dose fluoroscopic setting was applied for intraoperative imaging using a GE OEC 9800 c-arm for 34 patients and low-dose capture combined with real-time imageenhancement software (LessRay ${ }^{\circledR}$, NuVasive, Inc.) was used for 34 patients. The image-enhancement software also included surgical efficiency tools (e.g., tracking to reduce/eliminate scouting shots). All patients were treated in the lateral position for both interbody fusion and bilateral pedicle screw and rod fixation. Lateral (single position surgery) is defined as both anterior and posterior access to the spine without flipping the patient intraoperatively. Therefore, exclusion criteria would include anterior instrumentation or unilateral posterior fixation. Lateral interbody fusion was performed using either transpsoas lateral interbody fusion $\left(\mathrm{XLIF}^{\circledR}\right.$, NuVasive, Inc.) or lateral-position Anterior Lumbar Interbody Fusion (ALIF) approaches. Screws were placed percutaneously in all cases. Differences in radiation requirements between the two groups was determined by exposure time and mGy emission (Figure 1).

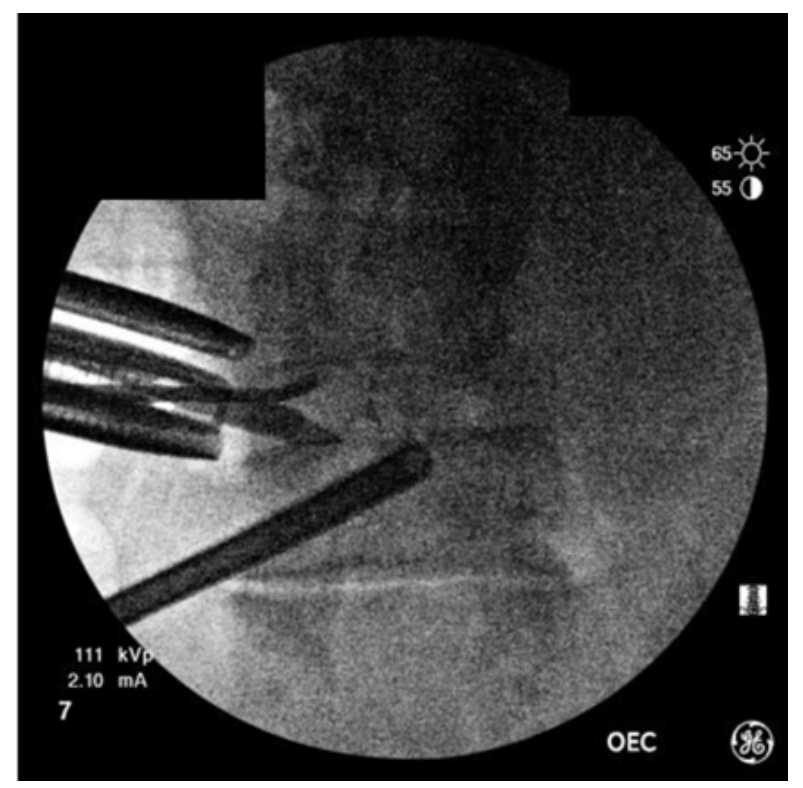

Figure 1: Low-dose pulse image in the AP plane during lateral interbody fusion. Note that the endplates and pedicles are difficult to identify appropriately. Risk of endplate injury, or aberrant screw placement is a concern. 
Of the 68 patients enrolled in the study, 15 (44.1\%) patients and $21(61.8 \%)$ patients had undergone MIS XLIF procedures in the image-enhanced group and standard low-dose group, respectively $(p=0.1449)$. In order to control for potential confounding variables such as sex, age and BMI independent $t$ test analysis was conducted using relevant collected health information. Because high crest or anterior-drifting psoas can often extend an L4-5 Lateral Lumber Interbody Fusion (LLIF) which may also account for increased radiation exposure in more complicated cases, operative levels were assessed between the groups for disparity. The specific levels treated in both dosing groups are presented in Table 1.

Table 1: Number of levels by radiation reduction technology.

\begin{tabular}{|c|c|c|c|}
\hline \multirow{2}{*}{ Levels } & \multicolumn{2}{|c|}{ Low-Dose Images } \\
\cline { 2 - 4 } & Standard low-dose & Image enhanced & Total \\
\hline L2-3 XLIF & 1 & 1 & 2 \\
\hline L2-4 XLIF & 2 & 1 & 5 \\
\hline L3-4 XLIF & 4 & 1 & 8 \\
\hline L3-5 XLIF & 6 & 2 & 10 \\
\hline L4-5 XLIF & 8 & 11 & 18 \\
\hline L5-S1 LALIF & 7 & 8 & 14 \\
\hline L5-S1 LALIF L4-5 XLIF & 6 & 34 & 68 \\
\hline
\end{tabular}

XLIF $=$ eXtreme lateral interbody fusion; LALIF = lateral anterior lumbar interbody fusion

Categorical data were compared using chi-squared tests, while continuous data gathered using F-tests. Significance was determined at $<0.01$. Sample size was not formally estimated for this observational study. However, the central limit theory suggests a sample size greater than 30 is adequate to assume normality, therefore it was estimated that the patient pool of 34 patients per group would be an accurate representation of the population and powered sufficiently to detect a significant mean difference between the groups, on the primary endpoints of radiation emission and fluoroscopic time.

\section{Results}

All cases were successfully performed with the patient maintained in the lateral position for both interbody fusion and bilateral pedicle screw fixation. There were no cases in either group that required revision of hardware for malpositioning. Intraoperative neuromonitoring was used for the placement of each pedicle screw. Due to the retrospective data collection, there was no missing data or follow-up failure so all 68 patients were included in analysis. Of those 68 cases 34 were preformed using ALARA dosing (standard low-dose group) while 34 were preformed using lowerdose radiation levels through image enhancement technology. The mean age of image enhanced group was 59.65 while the standard low-dose group was 57.26 (Table 2). Additionally, the mean BMI of image enhanced group was $30.37 \mathrm{~kg} / \mathrm{m}^{2}$ while the standard lowdose group was $29.55 \mathrm{~kg} / \mathrm{m}^{2}$ (Table 2). The image enhanced group contained 18 female participants while the standard low dose group contained 21 female participants (Table 2). None of these demographics (age, BMI, and sex) were found to be significantly different from each other $(p=0.4150, p=0.6050, p=0.4620$ respectively). The number of levels treated per procedure (singleor two-level) did not differ significantly across the dosing groups $(p=0.4505)$.

Table 2: Patient characteristics by imaging group.

\begin{tabular}{|c|c|c|c|}
\hline \multicolumn{5}{|c|}{ Low-Dose Images } \\
\hline Characteristic & Standard low-dose N=34 & Image enhanced N=34 & p-value \\
\hline Mean Age (year) (std) & $57.26(10.67)$ & $59.65(13.15)$ & 0.415 \\
Number of Females (\%) & $21(61.76)$ & $18(52.94)$ & 0.462 \\
Mean BMI (kg/m2) (std) & $29.55(6.75)$ & $30.37(6.20)$ & 0.605 \\
\hline Number of Levels & $20(58.82)$ & $23(67.65)$ & 0.4505 \\
Single (\%) & $14(41.18)$ & $11(32.35)$ & \\
Two (\%) & & & \\
\hline
\end{tabular}

Note: $\mathrm{std}=$ standard deviation $; \mathrm{kg} / \mathrm{m}=$ kilogram $/$ meters .

Mean total operative $\mathrm{C}$-arm radiation emission was found to be significantly lower in the image-enhanced group compared to the standard low-dose group (33.3mGy (standard deviation $\{\mathrm{std}\}$ : $18.4 \mathrm{mGy}$ ) vs. $52.2 \mathrm{mGy}$ (std: $36.8 \mathrm{mGy}$ ), respectively; $\mathrm{p}<0.01$ )(Table
3). Mean total fluoroscopy time was found to be significantly lower in the image-enhanced group compared to the standard low-dose group (110.6 seconds (std: 42.6 seconds) vs. 166.9 seconds (std: 51.9 seconds), respectively; $p<0.0001$ ) (Table 3 ). The effect of using 
intraoperative low-dose fluoroscopic images on both fluoroscopic radiation emission and fluoroscopy time remained a significant factor when controlled for age, sex, BMI, procedure (XLIF vs. ALIF), and number of levels (single- vs. two-level). These data show a
$36 \%$ reduction in radiation emission measured by $\mathrm{C}$-arm readouts in the image-enhanced cases, compared to standard ALARA-dose radiation cases. This reduction in radiation emission effect holds true when normalized across BMI, levels treated, and sex.

Table 3: Results.

\begin{tabular}{|c|c|c|c|}
\hline Radiation Imaging & \# of Cases & mGy Emission & Fluoroscopy Time (sec) \\
\hline Image-Enhanced ${ }^{\mathrm{a}, \mathrm{b}}$ & 34 & 33.3 & 110.6 \\
\hline ALARA dosing a,b & 34 & 52.2 & 166.9 \\
\hline \multicolumn{4}{|c|}{$\begin{array}{c}{ }^{a} \text { statistically significant difference }(\mathrm{p}<0.01) \text { indicating image-enhanced group showed less mGy emission than ALARA dosing (standard-low dose) } \\
\text { group. }\end{array}$} \\
\hline \multicolumn{4}{|c|}{$\begin{array}{l}{ }^{\mathrm{b}} \text { statistically significant difference }(\mathrm{p}<0.0001) \text { indicating image-enhanced group showed less fluoroscopy time than ALARA dosing (standard-low dose) } \\
\text { group. }\end{array}$} \\
\hline
\end{tabular}

To address the issue of type II error, a post hoc power assessment was conducted (nQuery, 2017) of the characteristics showing no mean difference between the groups: age and Body Mass Index (BMI) (Table 2). Assuming age differences above 65 and below 60 to be clinically meaningful, these estimates showed a sample size of 34 in each group would have $81 \%$ power to detect a difference in means of 7 (the difference between a Group 1 mean, $\mu_{1}$, of 66 and a Group 2 mean, $\mu_{2}$, of 59) assuming a common standard deviation of 10 using a two-group t-test or analysis of variance test with a $5 \%$ two-sided significance level. Assuming the difference between BMI scores at 25 (overweight) and 30 (Obese) to be clinically meaningful, a similar analysis estimated $98 \%$ power to detect this difference. Based on these results we concluded our sample was adequately powered (Figure 2).

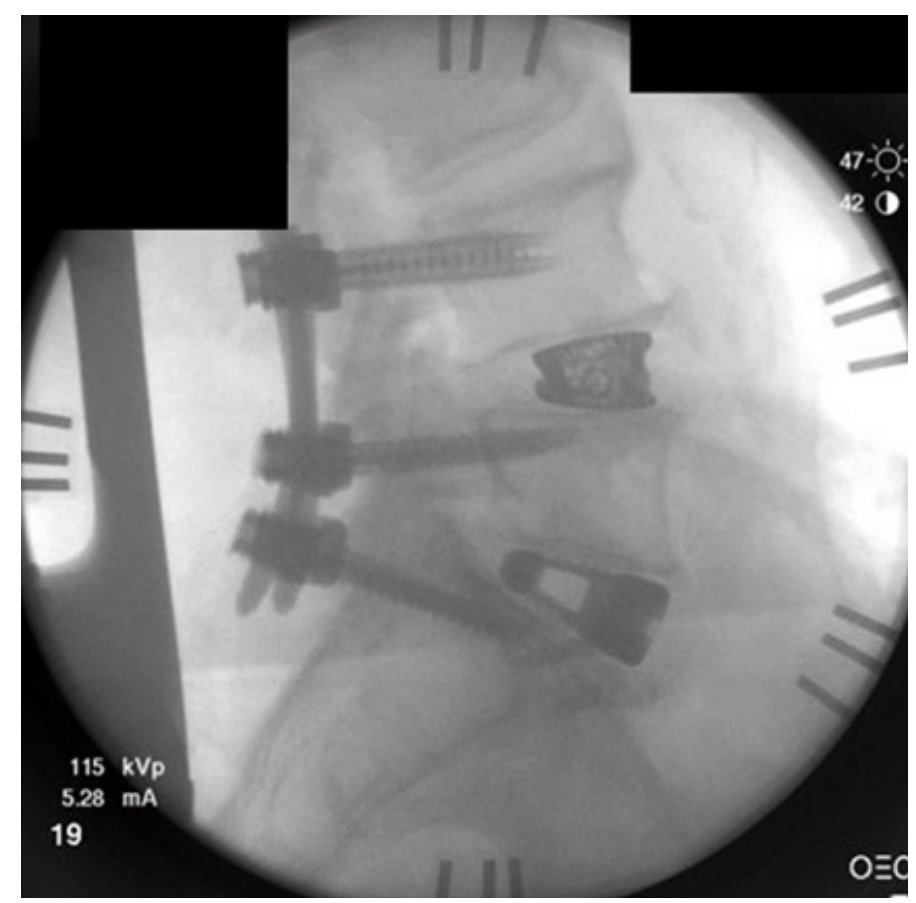

Figure 2: ALARA dosage plus image enhancement for a final intra-op pic after a two-level single, lateral-position lumbar fusion. All cortical structures necessary for intra-op evaluation and instrumentation are readily seen.

\section{Discussion}

ALARA is the guiding principle for radiation use and exposure, which indicates radiation exposure should be avoided at all costs unless it is deemed a relevant medical necessity. In the case of medical benefit, the exposure should be limited as much as possible while still providing adequate diagnostic utility. Since humans are exposed to passive radiation daily and that exposure compounds over time, reduced radiation exposure during surgical procedures is essential in order to further mitigate cancer, cataracts or other avoidable medical developments. Additionally while minimally invasive interventions have advantages over an open approach the need for imaging methods increases with decreasing invasiveness further adding to hospital personal exposure [5]. The FDA's center for Devices and Radiological Health launched an initiative in 2010 to reduce the unnecessary radiation exposure from medical imaging [6]. This two-part initiative demanded the need for justified reasoning and dose optimization, drawing attention to the 
medical necessity in lowering radiation doses through the use of modern techniques or the implementation of novel protocols. The continuation of research in radiation exposure to both patients and medical staff is crucial in the development of improved image quality. IET therefore, allows medical professionals to reduce the number of mAs required while ensuring ALARA standards are being met.

In the present study, indirect assessments of radiation dose (i.e., radiation emission and fluoroscopic time) associated with standard low-dose fluoroscopy and low-dose fluoroscopy with IET was prospectively examined during single-position lateral lumbar surgery. The results showed that the use of low-dose fluoroscopy with IET resulted in a $36 \%$ reduction in total radiation emission during XLIF and ALIF spinal procedures compared with the standard low-dose group. The current results are contiguous with previously reported reductions in radiation emission using ultralow-radiation imaging with LessRay IET [7]. While low-dose ALARA protocols have successfully reduced radiation exposure $[3,7,8]$, the results demonstrated that incorporating IET can further reduce radiation emission and potential radiation exposure of medical staff and patients during single-position lateral lumber surgery. Traditional ALIF procedures were noted to have significantly less radiation usage due to the nature of the workflow. Fluoroscopy shots were limited in ALIF procedures to localization of the operative site and final confirmation of anterior instrumentation. LLIF procedures uniformly required more radiation exposure due to the nature of the procedure. Flouroscopy was used routinely for passing instruments through the disc space for discectomy and endplate preparation as well as for placement of the interbody device.

Image quality remains to be a major concern for those who want to adopt low-dose fluoroscopy with IET protocols during spine surgery. In the first documented randomized clinical study on lowdose fluoroscopy, Wang et al. [7] found that ultra-low dose settings with IET can be utilized without compromising diagnostic utility of the images with consistent patient outcomes while reducing radiation emission by $75.5 \%$ during MIS TLIF [7]. In the current study, image quality was improved using lower-dose fluoroscopy with IET over the standard low-dose protocol. This was achieved with an emission reduction of approximately $36 \%$ from the standard low-dose protocol. Even-though the radiation emission achieved in this study using the low-dose protocol with IET (i.e., 33.3mGy) was slightly lower than emission values reported during MIS TLIF and LLIF, this appears to be due to the comparison in the current study between ALARA and ALARA with IET instead of standard-dose protocols compared to ALARA with IET [7,9]. Additionally, image quality may have been enhanced in the current study compared to prior work with IET, and this is likely due to continued refinements in IET protocols and incremental technological advancements that qualitatively yielded improved fluoroscopy images.

Furthermore, prolonged radiation exposure remains a reasonable concern for the patient and medical staff during MIS spine procedures. In the present study, fluoroscopic time was used as an indirect measurement of radiation exposure during lateral position surgery. The results of the current study showed that lowdose fluoroscopy with IET produced optimal image quality from a lower emission protocol while reducing total exposure time over standard ALARA protocol fluoroscopy during single-position lateral lumbar surgery. The use of a low-dose protocol with IET produced total exposure durations that were relatively consistent with prior studies that explored radiation exposure during a variety of spine procedures. For example, the total fluoroscopic time reported across spine surgery varies from 10.3 to 301 seconds for ACDF and kyphoplasty surgery, respectively [10]. Total fluoroscopic time for open TLIF and MIS TLIF was approximately 39 to 73 seconds, respectively [11]. For lateral approaches, total fluoroscopic time has been shown to be approximately 88.7 seconds [12]. While increases in total exposure time are generally related to more complicated procedures, surgeon's experience level, and patient specific attributes [7-12], the effect of prolonged radiation exposure can be can be offset and further mitigated in more complex procedures by utilizing low-dose fluoroscopy with IET in comparison to standard ALARA protocols While the amount of radiation emission is the product of milliamperes and exposure time [12], the results of the present study indicate that both radiation emission and fluoroscopic time can be reduced even further with lower-dose fluoroscopy with IET during lateral single-position surgery.

Studies on how protective measures reduce ionizing radiation exposure during MIS spine procedures have also been explored to gain a better understanding of associated risks to the surgeon and medical staff. In a study of radiation exposure, several vulnerable regions of the surgeon's body were identified through systematic placement dosimeters during LLIF surgery [13]. Taher et al. [12] found that regions of the body carried different vulnerabilities as the hands, axilla, eye, gluteal region, thyroid, and chest received on average $14.62,4.20,2.63,2.31,2.19$, and 0.44 mrem per LLIF case, respectively. When put into perspective longitudinally, Taher et al. [12] reported that it would take approximately 3420-6757 surgical cases to exceed the occupational radiation exposure limit when using ALARA protocol alone during LLIF surgery [12]. One major advantage of using low-dose fluoroscopy with IET is that the same number of MIS spine procedures can be performed with lower accumulated lifetime occupational exposure, which ultimately benefits surgeons, medical staff, and the patient.

It should be noted though that low-dose, ALARA protocols have been shown to be able to reduce the amount of radiation by up to a factor of 5 when compared to normal, high-dose protocols [14], and therefore are viable options in facilities where image enhancement is not available.

\section{Conclusion}

The goal of this study was to provide support to evaluate the utility of image-enhancing technologies, based on an initial scout $\mathrm{C}$-arm image, and to facilitate further radiation reduction intraoperatively than current unaided ALARA protocols. Furthermore, workflow advantages within the technology allow for the reduction of radiation by limiting the need for repetitive 
image capture (e.g., scouting). The results of this study suggest that applying IET on low-dose fluoroscopic images when performing single-position lateral lumbar surgery can reduce radiation emission, as well as fluoroscopic time, compared to low-dose protocol images, which results in reducing potential radiation exposure to the patient and medical staff during spine surgery.

\section{Acknowledgement}

The authors would like to acknowledge Kyle Malone, MS, Fatemeh Nooshabadi, PhD, and James Engall, PhD from NuVasive for editorial review of this manuscript.

\section{Conflicts of Interest}

Authors have completed the ICMJE uniform disclosure form. B. Braly is a consultant to NuVasive. However, Dr. Braly was not directly compensated for his or his team's work on this study or manuscript. The other authors have no conflicts of interest to declare. The authors have completed the STROBE reporting checklist.

\section{Ethical Statement}

The authors are accountable for all aspects of the work in ensuring that questions related to the accuracy or integrity of any part of the work are appropriately investigated and resolved. This retrospective, single-institution study was approved by the Western Institutional Review Board (IRB) (study \#281308) and consent was waived by the IRB for the retrospective chart review.

\section{References}

1. Mastrangelo G, Fedeli U, Fadda E, Giovanazzi A, Saia B, et Al. (2005) Increased cancer risk among surgeons in orthopaedic hospital. Occup Med 55(6): 498-500.

2. Rampersaud YR, FoleyKT, Shen AC, Williams S, Solomito M (2000) Radiation exposure to the spine surgeon during fluoroscopically assisted pedicle screw insertion. Spine 25(20): 2637-2645.

3. Bindal RK, Glaze S, Ognoskie M, Tunner V, Malone R, et al. (2008) Surgeon and patient radiation exposure in minimally invasive transforaminal lumbar interbody fusion. J Neurosurg Spine 9(6): 570-573.
4. Clark JC, Jasmer G, Marciano FF, Tumialán LM (2013) Minimally invasive transforaminal lumbar interbody fusions and fluoroscopy: a low-dose protocol to minimize ionizing radiation. Neurosurg Focus 35(2): E8.

5. Merter A, Karaeminogullari O, Shibayama M (2020) Comparison of radiation exposure among 3 different endoscopic diskectomy techniques for lumbar disk herniation. World Neurosurgery 139: 572-579.

6. (2019) Initiative to reduce unnecessary radiation exposure from medical imaging. U.s. Food \& Drug Administration.

7. Wang TY, Farber SH, Perkins SS, et al. (2017) Internally randomized control trial of radiation exposure using ultra-low radiation imaging versus traditional c-arm fluoroscopy for patients undergoing singlelevel minimally invasive transforaminal lumbar interbody fusion. Spine (Phila Pa 1976) 42(4): 217-223.

8. Ozgur BM, Aryan HE, Pimenta L, Taylor R (2006) Extreme Lateral Interbody Fusion (XLIF): a novel surgical technique for anterior lumbar interbody fusion. Spine J 6(4): 435-443.

9. Godzik J, Nayar G, Hunter WD, Tumialan LM (2019) Decreasing radiation emission in minimally invasive spine surgery using ultra-low-radiation imaging with image enhancement: A prospective cohort study. World Neurosurg 122: e805-e811.

10. Fransen P (2011) Fluoroscopic exposure in modern spinal surgery. Acta Orthop Belg 77: 386-389.

11. Wang J, Zhou Y, Zhang ZF (2011) Minimally invasive or open transforaminal lumbar interbody fusion as revision surgery for patients previously treated by open discectomy and decompression of the lumbar spine. Eur Spine J 20: 623-628.

12. Taher F, Hughes AP, Sama AA, Zeldin R, Schneider R, et al. (2013) Young Investigator Award winner: how safe is lateral lumbar interbody fusion for the surgeon? A prospective in vivo radiation exposure study. Spine (Phila Pa 1976) 38(16): 1386-1392.

13. Kukreja S, Haydel J, Nanda A, Sin AH (2015) Impact of body habitus on fluoroscopic radiation emission during minimally invasive spine surgery. J Neurosurg Spine 22(2): 211-218.

14. Pireau N, Cordemans V, Banse X, Irda N, Lichtherte S, et al. (2017) Radiation dose reduction in thoracic and lumbar spine instrumentation using navigation based on an intraoperative cone beam CT imaging system: a prospective randomized clinical trial. Eur Spine J 26(11): 2818-2827. 